\title{
Ksar Chebel (antique Petra), Ksar Adekkar (Kabylie)
}

Une route romaine fortifiée entre les vallées du Sebaou et de la Soummam

\section{J.-P. Laporte}

\section{OpenEdition}

\section{Journals}

Édition électronique

URL : http://journals.openedition.org/encyclopedieberbere/128

DOI : $10.4000 /$ encyclopedieberbere. 128

ISSN : 2262-7197

Éditeur

Peeters Publishers

\section{Édition imprimée}

Date de publication : 1 janvier 2008

Pagination : 4298-4300

ISBN : 2-7449-0707-4

ISSN : 1015-7344

Référence électronique

J.-P. Laporte, « Ksar Chebel (antique Petra), Ksar Adekkar (Kabylie)», Encyclopédie berbère [En ligne], 28-29 | 2008, document K75, mis en ligne le 01 juin 2013, consulté le 25 septembre 2020. URL : http:// journals.openedition.org/encyclopedieberbere/128 ; DOI : https://doi.org/10.4000/ encyclopedieberbere.128

Ce document a été généré automatiquement le 25 septembre 2020.

(c) Tous droits réservés 


\title{
Ksar Chebel (antique Petra), Ksar Adekkar (Kabylie)
}

\author{
Une route romaine fortifiée entre les vallées du Sebaou et de la \\ Soummam
}

J.-P. Laporte

1 Compte tenu de la difficulté du relief dans la région de Yakouren et de l'Akfadou, assurer la sécurité du passage entre la vallée du Sebaou et celle de la Soummam a de tout temps posé aux autorités du moment un redoutable problème. Autant, dans le reste de la Kabylie du Djurdjura, la plupart des «redoutes » et «fortins » romains signalés par les militaires français de la seconde moitié du XIX ${ }^{\mathrm{e}}$ siècle se sont révélés être de simples fermes, voire des huileries ou même des pressoirs isolés (Laporte 1980), on note dans ce secteur difficile une véritable route fortifiée romaine. Partant de Djemaa Saharidj (antique Bida), la voie antique descendait pour traverser le Sebaou, et remontait directement dans la ligne de plus grande pente vers Ifigha. Sur ce tronçon, on distingue à plusieurs reprises des vestiges d'empierrement, les deux lignes de gros blocs qui flanquaient de chaque côté la chaussée (de 5 à 6 mètres de large), les caniveaux qui la coupaient en oblique pour évacuer les eaux de ruissellement. Puis on arrivait sur un replat et, de là, à Ksar Chebel, où aboutissait également un diverticule venant de Rusazus (Azeffoun, ex-Port Gueydon). C'est à partir de là (et jusqu'à Ksar Kebbouch) que la voie était protégée par un dispositif exceptionnel. La route antique empruntait bientôt une crête, presque en ligne droite, jalonnée tous les un ou deux kilomètres par un poste de garde en maçonnerie de 7 mètres de côté. On arrivait ainsi jusqu'à une autre forteresse, Ksar Kebbouch, au-dessus du village d'Adekkar, dont on lui donne parfois le nom. Au-delà, en terrain plus aisé, la route se divisait en deux : une branche se dirigeait vers Saldae (Bejaia), par le Djebel Aghbalou («la montagne de la source ») et Toudja (d'où partait l'aqueduc alimentant la ville antique). Par l'autre branche (probablement la principale), on descendait directement et rapidement vers Tubusuptu (Tiklat, près d'El Kseur). Les deux forteresses ponctuaient donc fortement le dispositif, à deux endroits judicieusement choisis, à proximité immédiate d'un carrefour de la route principale avec un diverticule. Elles sont toutes deux construites en énormes pierres de taille. Ksar Chebel a été entièrement bouleversé à une époque 
ancienne, sans doute par un grand tremblement de terre, au point que l'on a du mal à en lever le plan (l'état du fort était déjà le même au temps de Vigneral 1868, p. 63). Le Ksar Kebbouch est mieux conservé, bien qu'un certain nombre de pierres aient été enlevées aux $\mathrm{XIX}^{\mathrm{e}}$ et $\mathrm{XX}^{\mathrm{e}}$ siècles pour élever le village d'Adekkar et le fameux « hôtel Lambert » tout proche. Nous avons pu lever en 1970 un plan (fig. 3) plus précis que celui donné par Mercier en 1885. En revanche, l'amas de pierres de taille et de moellons mêlés de Tala Kitane (Atlas, f. 6, $n^{\circ}$ 412) reste énigmatique.

Fig. 1 : Le profil de la route.

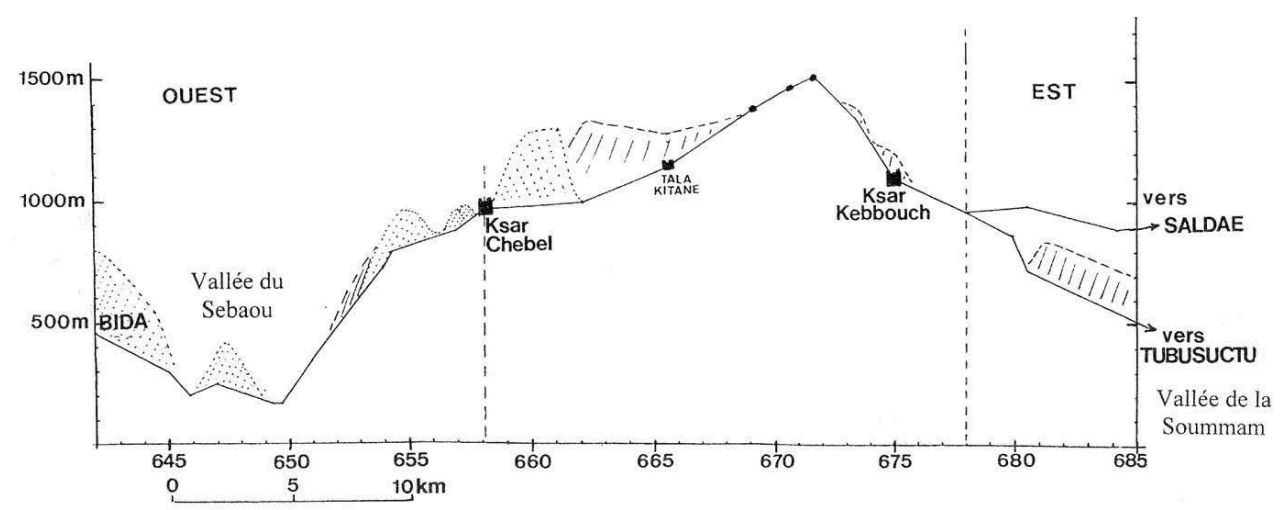

Dessin J.-P. Laporte.

2 Fig. 2 : Le site de Ksar Kebbouch vu du sud-est.

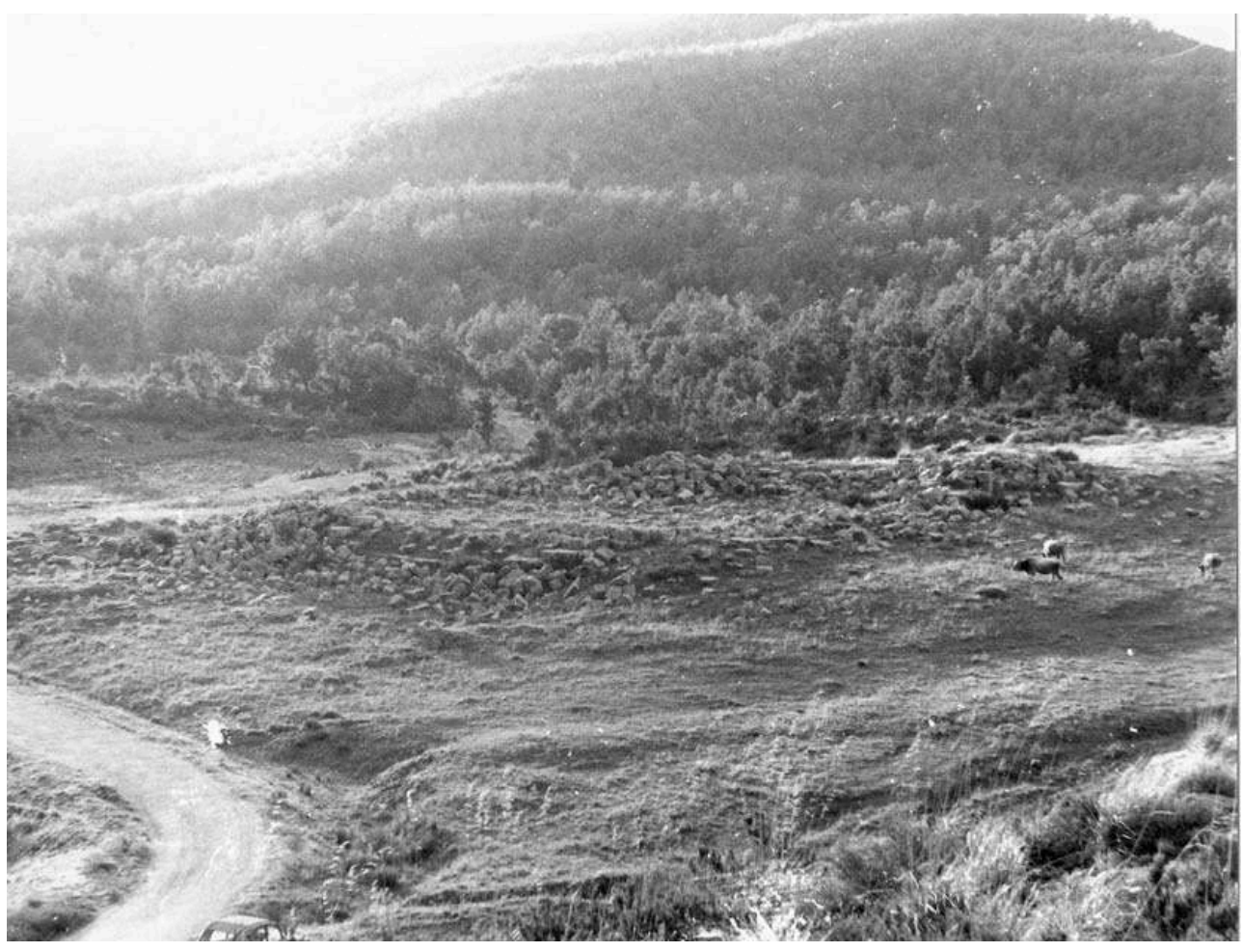

Cliché J.-P. Laporte 
Fig. 3 : Ksar Kebbouch.

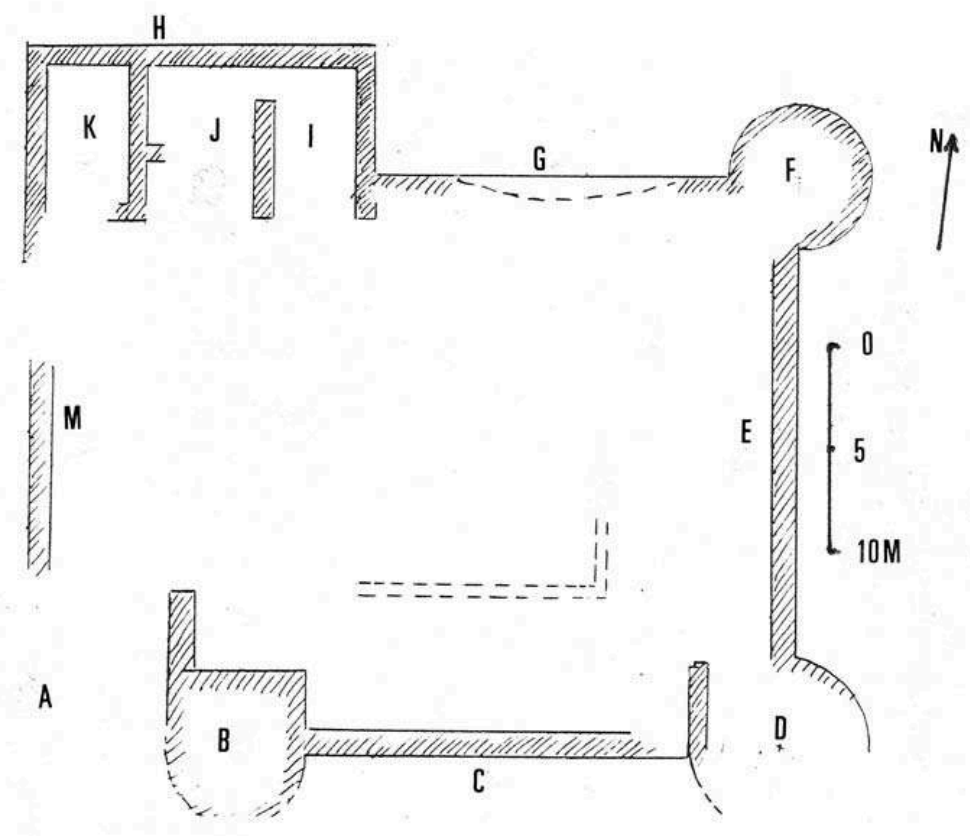

Plan J.-P. Laporte.

3 La route est certainement pré-romaine. Elle longe à plusieurs endroits des vestiges préet protohistoriques (notamment la pierre ornée de spirales du djebel Toukhra* et la grotte aux inscriptions libyques d'Ifigha*). En l'absence des dédicaces des deux forts (sans doute encore ensevelies sous les décombres), on peut approcher la date de son aménagement militaire romain. Il ne faut sans doute pas remonter à l'époque augustéenne, avec la fondation des trois colonies de Saldae (Bougie*), Tubusuctu (Tiklat, près El Kseur), etRusazus* (Azzefoun) (Laporte 1998). On peut envisager l'époque d'Hadrien, qui vit une intensification de l'occupation romaine dans la région. La route elle-même n'a pas à ce jour livré de milliaire dont la lecture puisse nous fixer. Les datations directes sont très rares. On n'en connaît guère qu'une stèle élevée à Ksar Chebel, sans doute vers la fin du II $^{\mathrm{e}}$ siècle et dédiée au Génie de Petra, le nom du lieu (à distinguer du Petra $^{*}$, situé dans la vallée de la Soummam, où Sammac* avait fait construire son château). En revanche, le diverticule qui conduit de Rusazus à Ksar Chebel a livré à Daouark la dédicace (entre 201 et 203, sous Septime Sévère) de la restauration d'une tour de surveillance (CIL,VIII, 8991), tombée en ruine (turrim e ruina lapsam), ainsi que deux milliaires de Philippe (244-249) et Claude II (268-270). On peut penser qu'elle a été rouverte sous Dioclétien, dans la mesure où un autre fort (celui d' Aqua Frigida, dont nous n'avons pas la description) a été restauré sous le praeses (gouverneur) Aurelius Litua, vers 290-293, sur une autre route de la région joignant Saldae à Sitifis. Quelque temps plus tard, c'est entre Ksar Chebel et Ksar Kebbouch que fut tracée la limite entre la Maurétanie césarienne et la Maurétanie sitifienne qui en fut détachée en 303 (Laporte 1996). La route n'était probablement plus défendue par l'armée romaine lors de la révolte de Firmus (370-373). En revanche, elle a sans doute été empruntée en 373 par les armées de Théodose l'Ancien (père de l'empereur) en 
route pour soumettre Firmus* (Laporte 2002, notamment, p. 291). Par la suite, son tracé, dicté par l'orographie, a sans doute été toujours emprunté par les voyageurs. Il le fut jusqu'à la création de la route moderne, tracée au prix d'importants travaux, un peu plus au nord, à flanc de montagne, beaucoup plus bas, et malgré tout parfois coupée l'hiver par la neige, coutumière en ce pays de montagne.

\section{BIBLIOGRAPHIE}

BAGHLI S. A. et FÉVRIER P.-A., « Recherches et travaux en 1967 », BAA, III, 1968, p. 1-34 p. 13-14 (stèle découverte par le P. J. Martin).

CARCOPINO J., « Note sur les antiquités du Tamgout d'Azazga », BCTH, 1919, p. 75-76, 172-173 et 1920, p. CXXII (au lieu dit Daouark).

GSELL St., Atlas archéologique de l'Algérie, f. 6, 1904 et addenda 1911, n 110 (Ksar Chebel) et $\mathrm{n}^{\circ} 115$ (Ksar Kebbouch), avec les fortins intermédiaires ( $\left.n^{\circ} 111-114\right) ; n^{\circ} 74$ (Daouark).

LAPORTE J.-P., « Fermes, huileries et pressoirs de Grande Kabylie », BCTH, n.s., 12-14, 1980, p. 131-157.

LAPORTE J.-P., « La Legio VII et la déduction des colonies augustéennes en Maurétanie césarienne », in Les Légions de Rome sous le Haut Empire, Colloque de Lyon, 1998, Lyon, 2000, p. 555-579.

LAPORTE J.-P., « Les armées romaines et la révolte de Firmus en Maurétanie césarienne », in L'armée romaine de Dioclétien à Valentinien ${ }^{I^{e r}}$, Congrès de Lyon, sept. 2002, Lyon, 2004, p. 279-298.

LAPORTE J.-P., « Une inscription de Saldae et la date de séparation des Maurétanies Césarienne et Sitifienne ", Africa romana, XII, 1996 (1998), p. 1111-1121.

MERCIER Lieutenant-colonel, « Notes sur les ruines et les voies antiques de l'Algérie recueillies par les soins des Brigades topographiques », BCTH, 1885, p. 354.

VIGNERAL Ch. de, Ruines romaines de l'Algérie. Kabylie du Djurdjura, 1868, p. 168 (Ksar Chebel) et p. 134-136 et 161 (Ksar Kebbouch).

\section{INDEX}

Mots-clés : Antiquité 